\title{
Necrosis del glande posterior a la colocación de un implante protésico peneano
}

\section{Necrosis of the glans penis after penile prosthesis implantation}

\author{
Walter Osvaldo De Bonis, ${ }^{1}$ Agustín Dangelo, ${ }^{2}$ Rodrigo Medel, ${ }^{3}$ Adolfo Casabe, ${ }^{4}$ Amado José Bechara ${ }^{5}$
}

\begin{abstract}
Resumen
ANTECEDENTES: La necrosis del glande, posterior a la colocación de un implante protésico peneano es una complicación extremadamente inusual. Las principales complicaciones se asocian con infección y falla mecánica del implante.

CASOS CLínIICOS: Se reportan dos casos de necrosis del glande, posterior a la colocación de implantes protésicos peneanos, uno con evolución satisfactoria, tratado de forma conservadora, sin necesidad de eliminar el implante y otro con mala evolución, que requirió la extracción de la prótesis y desbridamiento del tejido necrótico, con amputación espontánea del glande.

CONCLUSIONES: La necrosis del glande, posterior a la colocación de un implante protésico peneano, representa una complicación quirúrgica inusual, puede asociarse con elevada morbilidad y mortalidad, y suele afectar a pacientes con diabetes mal controlada o con mala vascularización preexistente.

PALABRAS CLAVE: Necrosis; glande; prótesis peneana; desbridamiento.

\section{Abstract}

BACKGROUND: Necrosis of the glans penis after the placement of a penile prosthetic implant is a very rare complication. The main complications are associated with infection and mechanical failure of the implant.

CLINICAL CASE: Two cases of glans necrosis occurring after penile prosthesis implantation are reported herein. One patient progressed satisfactorily with conservative treatment, with no need for implant removal. The other progressed poorly, requiring extraction of the prosthesis and debridement of the necrotic tissue. There was spontaneous amputation of the glans penis.

CONCLUSIONS: Necrosis of the glans penis after penile prosthesis implantation is a rare complication and can be associated with elevated morbidity and mortality. It tends to affect patients with uncontrolled diabetes or pre-existing poor vascularization.

KEYWORDS: Necrosis; Glans penis; Penile prosthesis; Debridement.
\end{abstract}

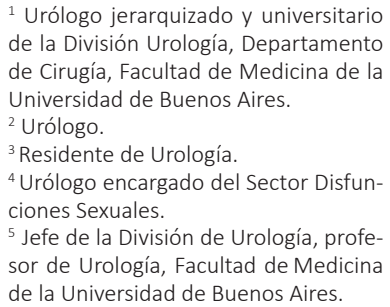

${ }^{1}$ Urólogo jerarquizado y universitario de la División Urología, Departamento de Cirugía, Facultad de Medicina de la Universidad de Buenos Aires.

${ }^{2}$ Urólogo.

${ }^{3}$ Residente de Urología.

${ }^{4}$ Urólogo encargado del Sector Disfunciones Sexuales.

${ }^{5}$ Jefe de la División de Urología, profesor de Urología, Facultad de Medicina de la Universidad de Buenos Aires.

Hospital Durand, Buenos Aires, Argentina.

Recibido: julio 2018

Aceptado: octubre 2018

Correspondencia

Walter Osvaldo De Bonis

deboniswalter@gmail.com

Instagram: walter_de_bonis

Este artículo debe citarse como

De Bonis WO, Dangelo A, Medel R, Casabe A, Bechara AJ. Necrosis del glande posterior a la colocación de un implante protésico peneano. Rev Mex Urol. 2018 nov-dic;78(6):447-51.

DOI: https://doi.org/10.24245/revmexurol.v78i6.2416

\section{ANTECEDENTES}

Desde hace más de cuatro décadas la colocación de un implante protésico peneano representa una opción eficaz y segura en pacientes con dis- función eréctil orgánica resistente al tratamiento médico. Las principales complicaciones relacionadas con esta práctica incluyen infección y falla mecánica del implante. La necrosis del glande posterior a la colocación de un implante 
protésico peneano es una complicación excepcional; pues existen pocos casos reportados en la bibliografía, que suponen un problema con alta morbilidad. El tratamiento consiste en extracción del implante, desbridamiento del tejido afectado, incluso glandectomía o penectomía parcial. Los casos publicados no muestran diferencias significativas en relación con el dispositivo empleado o el tratamiento con antibióticos prescritos. Los pacientes con este tipo de complicaciones suelen tener antecedentes de diabetes y aparece con mayor frecuencia en pacientes a quienes se practican cirugías subcoronales. Puesto que no existe bibliografía suficiente para establecer un consenso de tratamiento, aún se discute la conducta a seguir, ya sea conservadora o quirúrgica agresiva. A continuación se reportan dos casos de necrosis de glande posterior a la colocación de un implante peneano y se revisa la bibliográfica del tema.

\section{CASOS CLINICOS}

En ambos casos se siguió la preparación e indicación preoperatoria estandarizada para la cirugía del implante peneano, con aplicación de cefalotina en combinación con gentamicina en la inducción de la anestesia como tratamiento preventivo o de mantenimiento.

\section{Caso 1}

Paciente de 77 años, con antecedente de diabetes, no insulinodependiente; disfunción eréctil de 10 años de evolución, resistente al tratamiento con inhibidores de la fosfodiesterasa-5 (iPDE5) por vía oral y fármacos vasoactivos intracavernosos. En abril de 2018 se le implantó una prótesis maleable Spectra ${ }^{\circledR}$ (Boston Scientific, USA). En cuanto al procedimiento quirúrgico, se realizó una incisión subcoronal sin complicaciones, excepto una dilatación distal difícil del cuerpo cavernoso izquierdo. Cuatro horas después de la cirugía fue dado de alta del hospital con vendaje peneano no compresivo, sin catéter uretral. Al tercer día posoperatorio percibió ligera decoloración (oscura) perimeatal, con glande frío, pero sensibilidad conservada al estímulo táctil. En el séptimo día se observó casquete necrótico que afectaba por completo el hemiglande izquierdo y parte del hemiglande derecho, sin fiebre, leucocitosis, ni dificultad miccional. La ecografía Doppler evidenció flujo de arterias dorsales disminuido, ausencia de respuesta al estímulo doloroso y la prótesis en una posición normal. (Figura 1) Por la ausencia de signos clínicos de infección, se decidió mantener una conducta conservadora, continuando con el esquema antibiótico y aplicación local de Iruxol ${ }^{\circledR}$ (colagenasa, cloranfenicol; ungüento, AbbottEPD) dos veces al día. El paciente permaneció en seguimiento, con citas de control cada 72 h, espaciándose luego del primer mes posoperatorio, con lo que se observó

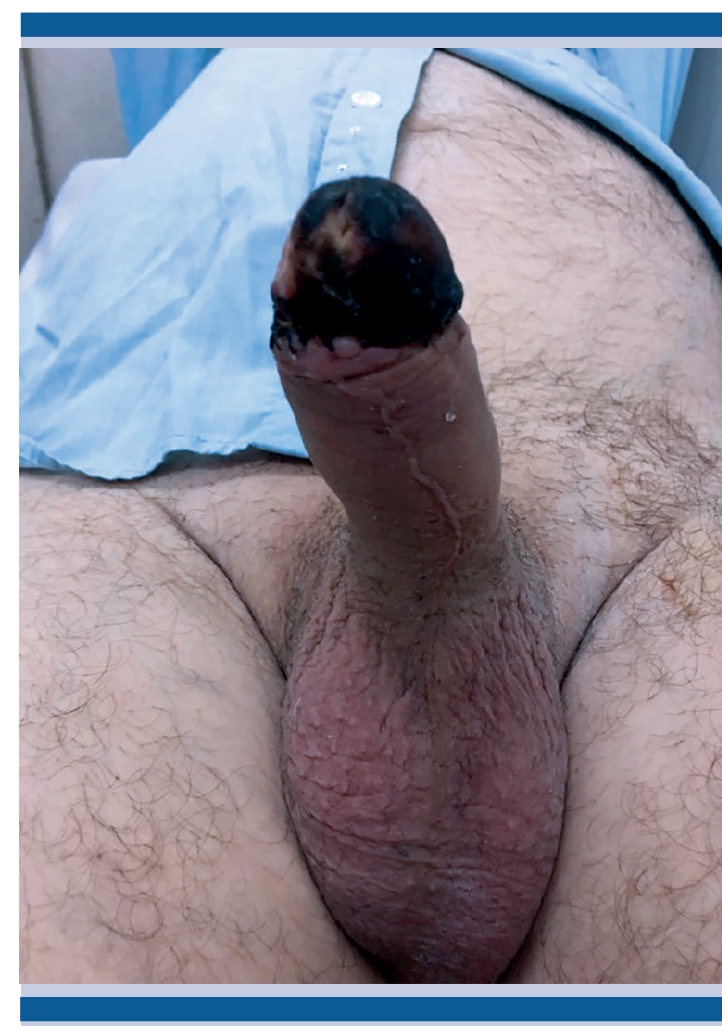

Figura 1. Necrosis seca (día 15 posoperatorio). Casquete necrótico con afectación casi total del glande, sin signos de infección. 
evolución satisfactoria y recuperación completa de la estructura tisular del glande. Se otorgó el alta médica a los 60 días de la cirugía. Figura 2

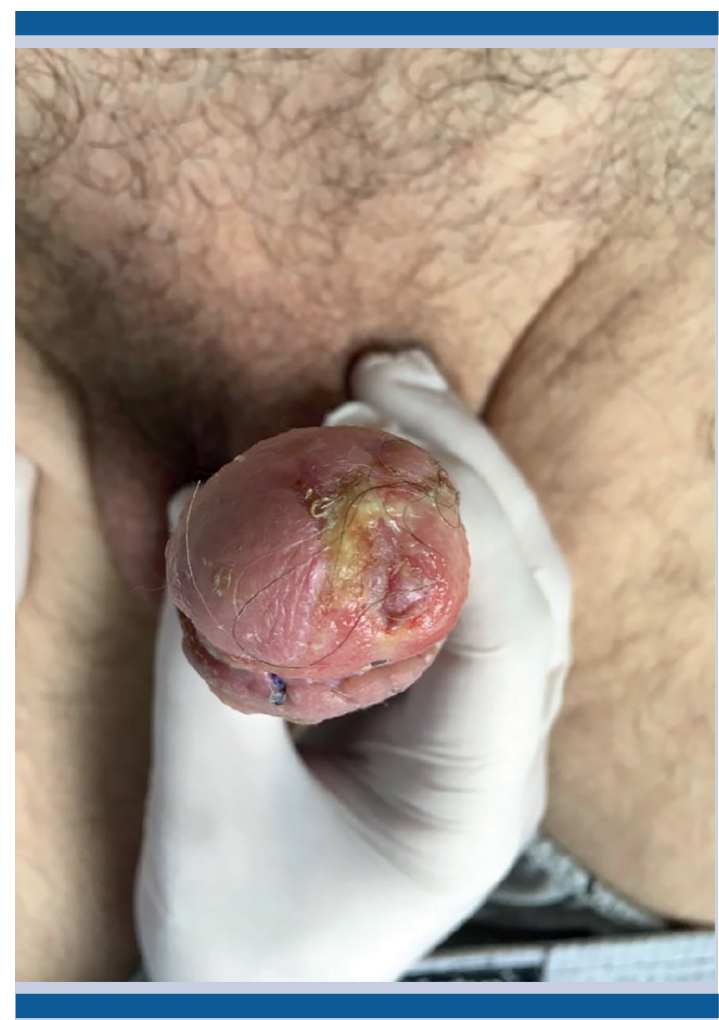

Figura 2. Necrosis seca (día 45 posoperatorio). Reepitelización de la mucosa con recuperación ad integrum de la estructura tisular.

\section{Caso 2}

Paciente de 61 años, con antecedentes de disfunción eréctil de varios años de evolución y curvatura peneana por enfermedad de De la Peyronie. En abril de 2018 se le colocó un implante protésico peneano Tube ${ }^{\circledR}$ (Promedon, Córdoba, Argentina) mediante incisión subcoronal, sin incidencias intraoperatorias, por lo que se otorgó el alta hospitalaria después de $4 \mathrm{~h}$ del procedimiento, sin colocación de catéter uretral y con apósitos en la herida quirúrgica no compresivos. A los 15 días del posoperatorio acudió a servicio médico por dolor, necrosis incipiente del glande, protrusión de los cilindros protésicos a nivel subcoronal, fiebre y exudado purulento (Figura 3). Se decidió la extracción del dispositivo y durante el procedimiento se apreció la salida de abundante material purulento de los cuerpos cavernosos, por lo que se prescribió tratamiento antibiótico de amplio espectro. Al día 30 del retiro del implante manifestó dificultad miccional y la formación de una fístula uretrocutánea ventral, con amputación parcial espontánea del glande. El tratamiento consistió en desbridamiento del tejido necrótico remanente y cistostomía suprapúbica (Figura 4). Se mantuvo en tratamiento con antibióticos y aplicación local de Iruxol ${ }^{\circledR}$. Sesenta días posteriores a la cirugía primaria se mantuvo la cistostomía, se observó tejido de granulación en la zona afectada, con amputación total del

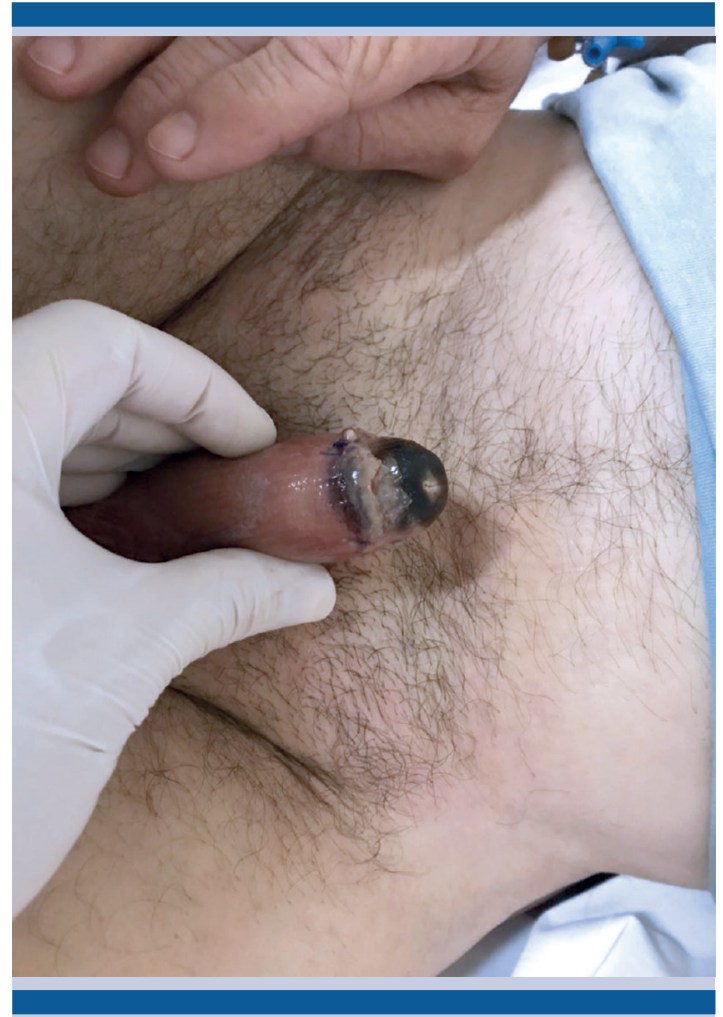

Figura 3. Necrosis húmeda del glande (día 15 posoperatorio), con exudado purulento en la línea de sutura quirúrgica. 


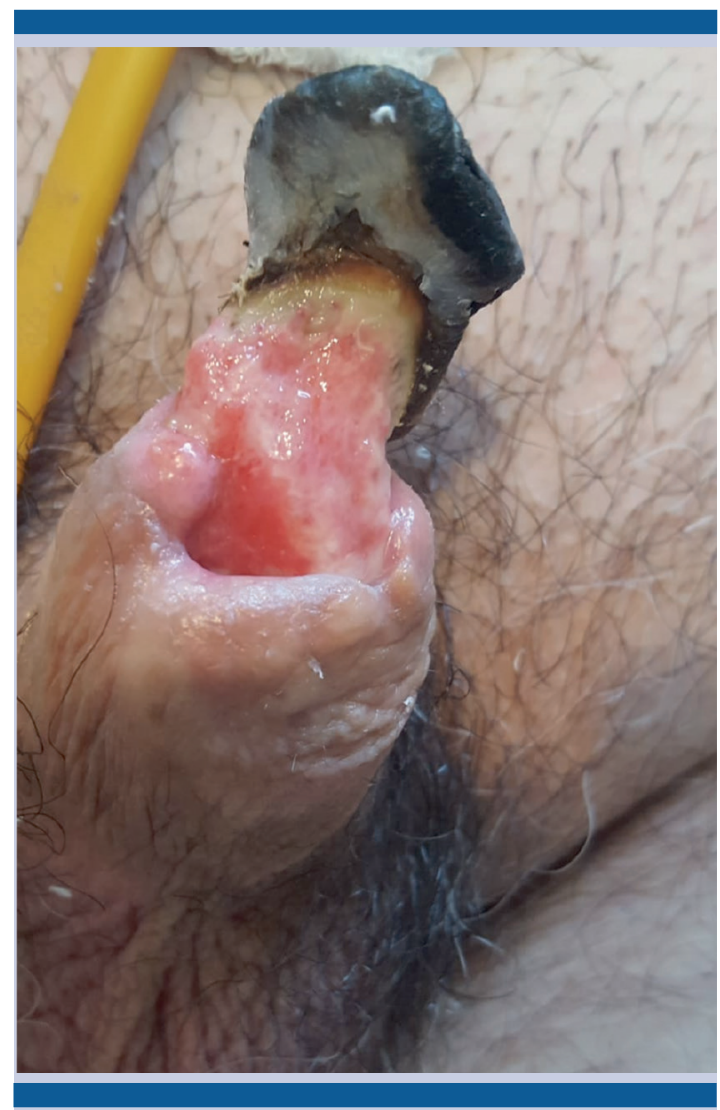

Figura 4. Necrosis húmeda (día 30 posoperatorio), con amputación espontánea del glande.

glande, planteándose eventual reconstrucción fálica y uretral en un primer tiempo.

\section{DISCUSIÓN}

La necrosis del glande puede clasificarse en seca o húmeda. La primera se atribuye a fenómenos isquémicos, sin requerimiento de tratamiento quirúrgico de urgencia; sin embargo, la necrosis húmeda o infecciosa, primaria o secundaria a sobreinfección por necrosis seca requiere intervención inmediata, porque puede provocar destrucción tisular progresiva y sepsis. ${ }^{1-3}$

La necrosis del pene, posterior a la colocación de un implante protésico, representa una com- plicación excepcional. Si bien existen diversos factores que contribuyen a este cuadro, las alteraciones vasculares preexistentes, la infección y las dilataciones traumáticas de los cuerpos cavernosos se consideran los principales elementos implicados en su origen. ${ }^{4}$ Incluso, se han descrito factores adicionales, que funcionan de forma individual o simultáneamente, como el vendaje compresivo o la colocación de catéter uretral. ${ }^{5}$ Sin embargo, de acuerdo con los casos reportados, el factor de riesgo más importante es la diabetes mellitus. ${ }^{6}$

Los conductos arteriales del pene provienen de la arteria pudenda interna, a través de sus ramas dorsal, cavernosa y bulbouretral, con abundante circulación colateral; no obstante, la circulación peneana, al igual que la coronaria, puede sufrir trastornos vasculopáticos sistemáticos. ${ }^{1-7}$

De acuerdo con algunos investigadores, la insuficiencia vascular preexistente puede exacerbarse por las dilataciones y el propio implante protésico al dañar e interrumpir el flujo cavernoso y generar presión de la vasculatura periférica por sobredistension, además de afectación vascular provocada por el vendaje compresivo. ${ }^{6}$ Chiang y sus colaboradores consideran que la colocación del implante, después de generar un proceso inflamatorio, provoca mayor requerimiento de oxígeno, nutrientes y mediadores inflamatorios, y al mismo tiempo aumento de la vasculatura peneana alterada, lo que resulta en un fenómeno isquémico. ${ }^{1}$

Los pocos casos reportados con esta complicación coinciden con los mismos tipos de prótesis (maleables e hidráulicas) y procedimientos subcoronales. Hasta la fecha no se han observado diferencias en la prescripción de antibióticos combinados con la inducción preanestésica, ni terapéutica de mantenimiento.

Si bien existe consenso en que las infecciones protésicas ocurren frecuentemente en pacientes 
con diabetes mal controlada, Minervini y su grupo señalan que no existe correlación entre las concentraciones elevadas de hemoglobina glucosilada e infección, incluso con mayor incidencia de necrosis. ${ }^{8}$

Por el momento no existe un consenso o protocolo de tratamiento para esta alteración; ${ }^{9} \sin$ embargo, se sugiere una conducta conservadora en pacientes con necrosis, que no manifiestan signos clínicos o infección. ${ }^{7}$ Weiner y su equipo de trabajo sugieren que la intervención quirúrgica temprana puede prevenir la evolución a necrosis húmeda. ${ }^{10}$ Uno de los casos expuestos en este estudio demostró que la vigilancia activa, el tratamiento con antibiótico de mantenimiento y el tratamiento local de la necrosis permitieron conservar el implante y la regeneración del tejido afectado. En pacientes con necrosis e infección clínica se sugiere una conducta quirúrgica agresiva, con desbridamiento del tejido afectado y extracción de la prótesis.

\section{CONCLUSIÓN}

La necrosis del glande posterior a la colocación de un implante protésico peneano representa una complicación quirúrgica inusual, puede asociarse con elevada morbilidad y mortalidad, y suele afectar a pacientes con diabetes mal controlada o con vascularización preexistente. Cuando la necrosis aparece con signos de infección, el tratamiento inicial consiste en extracción de la prótesis y desbridamiento del tejido afecta- do; sin embargo, en pacientes con necrosis local, sin signos de actividad o mala evolución, debe intentarse una conducta conservadora.

\section{REFERENCIAS}

1. Chiang IN, et al. Management of ischemic penile gangrene: prompt partial penectomy and other treatment options. J Sex Med 2008;5:2725-2733. DOI: 10.1111/j.17436109.2008.00894.x

2. Harris $\mathrm{CF}, \mathrm{Mydlo} \mathrm{JH}$. Ischemia and gangrene of the penis. J Urol 2003;169(5):1795. DOI: 10.1097/01. ju.0000057796.64080.3a

3. Wen CC, et al. Management of ischemic priapism with high-dose intracavernosal phenylephrine: From bench to bedside. J Sex Med 2006;3:918-22. DOI: 10.1111/j.17436109.2005.00140.x

4. Shelling RH, Maxted WC. Major complications of silicone penile prosthesis Predisposing clinical situations. Urology 1980;15(2):131-3.

5. Kardar A, Pettersson BA. Penile gangrene: a complication of penile prosthesis. Scand J Urol Nephrol 1995;29:355-356.

6. Darwich B, et al. Gangrene of the penis after implantation of penile prosthesis: Case reports, treatment recommendations and review of the literature. J Urol 1993;150(1):190-192.

7. El-Sakka Al, et al. Coronary artery risk factors in patients with erectile dysfunction. J Urol 2004;172:251-4. DOI: 10.1097/01.ju.0000128572.31000.f0

8. Minervini A, et al. Outcome of penile prosthesis implantation for treating erectile dysfunction: experience with 504 procedures. BJU Int 2006;97(1):129-133. DOI: 10.1111/j.1464-410X.2005.05907.x

9. García-Gómez B, et al. Ischemic gangrene of the glans following penile prosthesis implantation. Case Rep Urol 2013;2013:323574. DOI: 10.1155/2013/323574

10. Weiner DM, Lowe FC. Surgical management of ischemic penile gangrene in diabetics with end stage atherosclerosis. J Urol1996;155:926-9. 